\title{
Uniform Asymptotic Stability and Global Asymptotic Stability for Time-Delay Hopfield Neural Networks
}

\author{
Adnene Arbi, Chaouki Aouiti, and Abderrahmane Touati \\ University of Carthage, Faculty of sciences of Bizerta, Department of Mathematics, \\ 7021 Jarzouna Bizerta, Tunisia \\ adnen.arbi@enseignant.edunet.tn, \{chaouki.aouiti,Abder.Touati\}@fsb.rnu.tn
}

\begin{abstract}
In this paper, we consider the uniform asymptotic stability and global asymptotic stability of the equilibrium point for time-delays Hopfield neural networks. Some new criteria of the system are derived by using the Lyapunov functional method and the linear matrix inequality approach for estimating the upper bound of the derivative of Lyapunov functional. Finally, we illustrate a numerical example showing the effectiveness of our theoretical results.
\end{abstract}

\section{Introduction}

Hopfield ([1, 2] has proposed Hopfield neural networks (HNN) which have found applications in a broad range of discipline where the targeted problems can reduce to optimization problems. It has been extensively studied and developed in recent years, and it has attracted much attention in the literature on Hopfield neural networks with time delays,. They are now recognized as candidates for information processing systems and have been successfully applied to associative memory, pattern recognition, automatic control, model identification, optimization problems, etc. (we refer to reader 3[10). Therefore, the study of stability of HNN has caught many researchers' attention. HNN with time delays has been extensively investigated over the years, and various sufficient conditions for the stability of the equilibrium point of such neural networks have been presented via different approaches. In [5], 13], some sufficient conditions of stability by utilizing the Lyapunov functional method, and linear matrix inequality approach for delayed continuous HNN are derived. In 14, G.Zong and J.Liu established a novel delay-dependent condition to guarantee the existence of HNN and its global asymptotic stability by resorting to the integral inequality and constructing a Lyapunov-Krasovskii functional. In [16, S.Long and D.Xu got the sufficient conditions for global exponential stability and global asymptotic stability by using Lyapunov-Krasovskii-type functionally of negative definite matrix and Cauchy criterion.

This paper is organized as follows: In section 2, a model of time-delay Hopfield neural network is described. In addition, we present some basic definitions and lemmas. New stability criteria for Hopfield neural network are derived in section

L. Iliadis et al. (Eds.): AIAI 2012, IFIP AICT 381, pp. 483-492, 2012.

(C) IFIP International Federation for Information Processing 2012 
3. An example is given in section 4, to illustrate the advantage of the results obtained. Finally, some conclusions are drawn in section 5 .

\section{Preliminaries}

Let $\mathbb{R}$ denote the set of real numbers, $\mathbb{Z}_{+}$denote the positive integers and $\mathbb{R}^{n}$ denote the $n$-dimensional real space equipped with the Euclidean norm $\|$.$\| . The$ identity matrix, with appropriate dimensions, is denoted by $I d$ and $\operatorname{diag}(\ldots)$ denotes the block diagonal matrix.

Consider the following delayed HNN model with impulses

$$
\left\{\begin{array}{l}
\dot{x}_{i}(t)=-c_{i} x_{i}(t)+\sum_{j=1}^{n} a_{i j} f_{j}\left(x_{j}(t)\right) \\
+\sum_{j=1}^{n} b_{i j} g_{j}\left(x_{j}(t-\tau(t))\right)+I_{i} \text { if } t \neq t_{k} \\
\triangle x_{i} \backslash t=t_{k}=d_{k}^{(i)}\left(x_{i}\left(t_{k}^{-}\right)-\bar{x}_{i}\right) \quad i=1, \ldots, n, \quad n, k \in \mathbb{Z}_{+},
\end{array}\right.
$$

where $n \geq 2$ corresponds to the number of units in a neural network; the impulsive times $t_{k}$ satisfy:

$0 \leq t_{0}<t_{1}<\ldots<t_{k}<\ldots, \lim _{k \rightarrow+\infty} t_{k}=+\infty ; x_{i}$ corresponds to the state of the unit $i$ at time $t ; c_{i}$ is positive constant; $f_{j}, g_{j}$, denote respectively, the measures of response or activation to their incoming potentials of the unit $j$ at time $t$ and $t-\tau(t)$; constant $a_{i j}$ denotes the synaptic connection weight of the unit $j$ on the unit $i$ at time $t$; constant $b_{i j}$ denotes the synaptic connection weight of the unit $j$ on the unit $i$ at time $t-\tau(t) ; I_{i}$ is the input of the unit $i ; \tau(t)$ is the transmission delay such as $0<\tau(t) \leq \tau$ and $\dot{\tau}(t) \leq \rho<1 ; t \geq t_{0} ; \tau, \rho, d_{k}^{(i)}$ are constants; $\bar{x}$ is the equilibrium point of the first equation in the system (1). The evolution of the neuron state $i$ at time $t_{k}$ is described by the equation:

$$
\triangle x_{i} \backslash_{t=t_{k}}=x_{i}\left(t_{k}\right)-x_{i}\left(t_{k}^{-}\right)=d_{k}^{(i)}\left(x_{i}\left(t_{k}^{-}\right)-\bar{x}_{i}\right), i=1,2, \ldots, n, k=1,2, \ldots
$$

The initial conditions associated with system (1) are of the form:

$$
x(s)=\phi(s), s \in\left[t_{0}-\tau, t_{0}\right]
$$

where

$$
x(s)=\left(x_{1}(s), x_{2}(s), \ldots, x_{n}(s)\right)^{T}, \phi(s)=\left(\phi_{1}(s), \phi_{2}(s), \ldots, \phi_{n}(s)\right)^{T} \in P C\left([-\tau, 0], \mathbb{R}^{n}\right)
$$

$P C\left([-\tau, 0], \mathbb{R}^{n}\right)=\left\{\psi:[-\tau, 0] \longrightarrow \mathbb{R}^{n}\right.$, is continuous everywhere except at finite number of points $t_{k}$, at which $\psi\left(t_{k}^{+}\right)$and $\psi\left(t_{k}^{-}\right)$exist and $\left.\psi\left(t_{k}^{+}\right)=\psi\left(t_{k}\right)\right\}$. For $\psi \in P C\left([-\tau, 0], \mathbb{R}^{n}\right)$, the norm of $\psi$ is defined by

$$
\|\psi\|_{\tau}=\sup _{-\tau \leq \theta \leq 0}\|\psi(\theta)\|
$$

For any $t_{0} \geq 0$, let: $P C_{\delta}\left(t_{0}\right)=\left\{\psi \in P C\left([-\tau, 0], \mathbb{R}^{n}\right):\|\psi\|_{\tau}<\delta\right\}$. 
In this paper, we assume that some conditions are satisfied so that the equilibrium point of system (10) does exist, see ([5], 11]). Impulsive operator is viewed as perturbation of the equilibrium point $\bar{x}$ of such system without impulsive effects.

Since $\bar{x}$ is an equilibrium point of system (1), one can derive from system (1) that the transformation $y_{i}=x_{i}-\bar{x}_{i}, i=1,2, . . n$ transforms such system into the following system:

$$
\left\{\begin{array}{l}
\dot{y}_{i}(t)=-c_{i} y_{i}(t)+\sum_{j=1}^{n} a_{i j} F_{j}\left(y_{j}(t)\right)+\sum_{j=1}^{n} b_{i j} G_{j}\left(y_{j}(t-\tau(t))\right) \text { si } t \neq t_{k} \\
y_{i}\left(t_{k}\right)=\left(1+d_{k}^{(i)}\right) y_{i}\left(t_{k}^{-}\right) \quad i=1, \ldots, n, \quad n, k \in \mathbb{Z}_{+},
\end{array}\right.
$$

where $F_{j}\left(y_{j}(t)\right)=f_{j}\left(\bar{x}_{j}+y_{j}(t)\right)-f_{j}\left(\bar{x}_{j}\right)$ and $G_{j}\left(y_{j}(t-\tau(t))\right)=g_{j}\left(\bar{x}_{j}+y_{j}(t-\tau(t))\right)-g_{j}\left(\bar{x}_{j}\right)$.

To prove the stability of $\bar{x}$ of system (1), it is sufficient to prove the stability of the zero solution of system (3).

In this paper, we assume that there exist constants $L_{i}, M_{i} \geq 0$ such as

$$
\left|F_{i}(y)\right| \leq L_{i}|y|,\left|G_{i}(y)\right| \leq M_{i}|y|, i \in \Lambda=\{1,2, \ldots n\},
$$

and we set

$$
\begin{aligned}
& L_{\max }=\max _{i \in \Lambda} L_{i}, M_{\max }=\max _{i \in \Lambda} M_{i}, c_{\max }=\max _{i \in \Lambda} c_{i}, \\
& c_{\min }=\min _{i \in \Lambda} c_{i}, D_{k}=\operatorname{diag}\left(1+d_{k}^{(1)}, 1+d_{k}^{(2)}, \ldots, 1+d_{k}^{(n)}\right) .
\end{aligned}
$$

So, the system (3) can be written as follows:

$$
\left\{\begin{array}{l}
\dot{y}(t)=-C y(t)+A \cdot F(y(t))+B \cdot G(y(t-\tau(t))) \text { si } t \neq t_{k} \\
y\left(t_{k}\right)=D_{k} y\left(t_{k}^{-}\right) \quad i=1, \ldots, n, \quad n, k \in \mathbb{Z}_{+}, \\
y\left(t_{0}+\theta\right)=\varphi(\theta),
\end{array}\right.
$$

where

$$
\begin{aligned}
& y(t)=\left(y_{1}(t), \ldots, y_{n}(t)\right)^{T} ; y(t-\tau(t))=\left(y_{1}(t-\tau(t)), \ldots, y_{n}(t-\tau(t))\right)^{T} ; \\
& C=\operatorname{diag}\left[c_{1}, \ldots, c_{n}\right] ; A=\left(a_{i j}\right)_{n \times n} ; B=\left(b_{i j}\right)_{n \times n} ; F(y)=\left(F_{1}\left(y_{1}\right), F_{2}\left(y_{2}\right), \ldots, F_{n}\left(y_{n}\right)\right)^{T} ; \\
& G(y)=\left(G_{1}\left(y_{1}\right), G_{2}\left(y_{2}\right), \ldots, G_{n}\left(y_{n}\right)\right)^{T} .
\end{aligned}
$$

Some definitions and lemma of stability for system (11) at its equilibrium point are introduced as follows:

Definitions 21. Assume $y(t)=y\left(t_{0}, \varphi\right)(t)$ be the solution of (3) through $\left(t_{0}, \varphi\right)$, then the zero solution of (3) is said to be [12] 
$\mathbf{P 1}$ stable, if for any $\epsilon>0$ and $t_{0} \geq 0$, there exists some $\delta\left(\epsilon, t_{0}\right)>0$ such as $\varphi \in P C_{\delta}\left(t_{0}\right)$ implies $\left\|y\left(t_{0}, \varphi\right)(t)\right\|<\epsilon, \quad t \geq t_{0}$.

$\mathbf{P 2}$ uniformly stable, if the $\delta$ in (P1) is independent of $t_{0}$.

P3 uniformly attractive, if there exists some $\delta>0$ such as for any $\epsilon>0$, there exists some $T=T(\epsilon, \delta)>0$ such as $t_{0} \geq 0$ and $\varphi \in P C_{\delta}\left(t_{0}\right)$ implies $\left\|y\left(t_{0}, \varphi\right)(t)\right\|<\epsilon, \quad t \geq t_{0}+T$.

$\mathbf{P} 4$ uniformly asymptotically stable, if (P2) and (P3) are held.

P5 globally asymptotically stable, if (P1) holds and for any given initial value $y_{0}=\varphi,\left\|y\left(t_{0}, \varphi\right)(t)\right\| \longrightarrow 0$ as $t \longrightarrow+\infty$.

Now, we need the following basic lemmas used in our work.

Lemma 21. [15] For any $a, b \in \mathbb{R}^{n}$, the inequality

$$
\pm 2 a^{T} b \leq a^{T} X a+b^{T} X^{-1} b
$$

holds, where $X$ is any $n \times n$ matrix with $X>0$.

\section{Robust Stability Criteria}

In this section, we shall establish some theorems which provide sufficient conditions for uniform asymptotic stability and global asymptotic stability of system (11).

Theorem 31. The system (1) is uniformly stable if there is $\epsilon^{*} \in[0,1], \sigma>0$ and positive $n \times n$ definite matrix $Q$ such as:

(i) $\sigma \cdot \frac{\max _{i, j} c_{i} \cdot q_{i j}}{\min _{i, j}\left|b_{i j}\right|} \leq \frac{\tau^{2}+4-\tau \sqrt{\tau^{2}+4}}{\tau^{2}+4+\tau \sqrt{\tau^{2}+4}}$

(ii) $\max _{1 \leq i \leq n}\left\{\frac{1}{c_{i}} \sum_{j=1}^{n} a_{i j}\right\}+\max _{1 \leq j \leq n}\left\{L_{j}^{2} \sum_{i=1}^{n} \frac{a_{i j}}{c_{i}}\right\}+\max _{1 \leq j \leq n}\left\{M_{j}^{2} \sum_{i=1}^{n} \frac{\left|b_{i j}\right|}{c_{i}}\right\}+\frac{\epsilon^{*}}{c_{m i n}}$

$+\frac{1}{\sigma} \lambda_{\max }\left(C^{-1} B Q^{-1} B^{T} C^{-1}\right)<2$

(iii) $\frac{\prod_{0}<t_{k}<t}{1+\epsilon^{*}\left(t-t_{0}\right)^{2}}<\infty$, where $\xi_{k}$ is the largest eigenvalue of $D_{k} C^{-1} D_{k}$, and $k \in \mathbb{Z}_{+}$.

In addition, if we have:

(iv) $\frac{\prod_{t_{0}<t_{k}<t} \max \left\{c_{\max } \xi_{k}, 1\right\}}{1+\epsilon^{*}\left(t-t_{0}\right)^{2}} \longrightarrow 0$, if $t \longrightarrow+\infty$, then the system (11) is uniformly asymptotically stable and globally asymptotically stable.

Proof. First, we prove the equilibrium point of system (11) is uniformly stable. We consider this Lyapunov function:

$$
V(y)(t)=\left[1+\epsilon^{*}\left(t-t_{0}\right)^{2}\right] \sum_{i=1}^{n} \frac{1}{c_{i}} y_{i}^{2}(t)+\sum_{i=1}^{n} \sum_{j=1}^{n} \frac{\left|b_{i j}\right|}{c_{i}} \int_{t-\tau(t)}^{t}\left(1+\left(s-t_{0}\right)^{2}\right) G_{j}^{2}\left(y_{j}(s)\right) d s .
$$


For condition (iii), there is a constant $M^{*}>0$, such as:

$$
\frac{\prod_{t_{0}<t_{k}<t} \max \left\{c_{\max } \xi_{k}, 1\right\}}{1+\epsilon^{*}\left(t-t_{0}\right)^{2}}<M^{*}, t \geq t_{0} .
$$

For any $t_{0} \geq 0$, let $y\left(t_{0}, \varphi\right)(t)$ be a solution of system (1). So, $\forall \epsilon>0$, we choose $\delta$ of the following manner:

$$
\delta=\sqrt{\frac{1}{c_{\max }\left[\frac{1}{c_{\min }}+\max _{1 \leq i \leq n}\left\{\sum_{j=1}^{n} M_{j}^{2} \frac{\left|b_{i j}\right|}{c_{i}}\right\}\left(\tau+\frac{\tau^{3}}{3}\right)\right] M^{*}}} . \epsilon
$$

By simple calculation:

$$
\begin{aligned}
& \frac{\partial V(y)(t)}{\partial t}=\left[1+\epsilon^{*}\left(t-t_{0}\right)^{2}\right]\left\{-2 \sum_{i=1}^{n} y_{i}^{2}(t)+2 \sum_{i=1}^{n} \sum_{j=1}^{n} \frac{a_{i j}}{c_{i}} y_{i}(t) F_{j}\left(y_{j}(t)\right)\right. \\
&\left.+2 \sum_{i=1}^{n} \sum_{j=1}^{n} \frac{b_{i j}}{c_{i}} y_{i}(t) G_{j}\left(y_{j}(t-\tau(t))\right)\right\}+2 \epsilon^{*}\left(t-t_{0}\right) \sum_{i=1}^{n} \frac{1}{c_{i}} y_{i}^{2}(t) \\
&+\sum_{i=1}^{n} \sum_{j=1}^{n} \frac{\left|b_{i j}\right|}{c_{i}}\left(1+\left(t-t_{0}\right)^{2}\right) G_{j}^{2}\left(y_{j}(t)\right)-\sum_{i=1}^{n} \sum_{j=1}^{n} \frac{\left|b_{i j}\right|}{c_{i}}\left(1+\left(t-\tau(t)-t_{0}\right)^{2}\right) G_{j}^{2}\left(y_{j}(t-\tau(t))\right) .
\end{aligned}
$$

Therefore,

$$
\begin{gathered}
\frac{\partial V(y)(t)}{\partial t}=-2\left(1+\epsilon^{*}\left(t-t_{0}\right)^{2}\right) \sum_{i=1}^{n} y_{i}^{2}(t)+\left(1+\epsilon^{*}\left(t-t_{0}\right)^{2}\right)\left\{\sum_{i=1}^{n} \sum_{j=1}^{n} \frac{a_{i j}}{c_{i}} y_{i}^{2}(t)\right. \\
\left.+\sum_{i=1}^{n} \sum_{j=1}^{n} \frac{a_{i j}}{c_{i}} F_{j}^{2}\left(y_{j}(t)\right)+\frac{1}{\sigma} \lambda_{\max }\left(C^{-1} B Q^{-1} B^{T} C^{-1}\right) \sum_{i=1}^{n} y_{i}^{2}(t)\right\} \\
+2 \epsilon^{*}\left(t-t_{0}\right) \sum_{i=1}^{n} \frac{1}{c_{i}} y_{i}^{2}(t)+\sigma\left(1+\epsilon^{*}\left(t-t_{0}\right)^{2}\right) \sum_{i=1}^{n} \sum_{j=1}^{n} q_{i j} G_{j}^{2}\left(y_{j}(t-\tau(t))\right) \\
-\left(1+\left(t-\tau(t)-t_{0}\right)^{2}\right) \sum_{i=1}^{n} \sum_{j=1}^{n} \frac{\left|b_{i j}\right|}{c_{i}} G_{j}^{2}\left(y_{j}(t-\tau(t))\right)+\left(1+\left(t-t_{0}\right)^{2}\right) \sum_{i=1}^{n} \sum_{j=1}^{n} \frac{\left|b_{i j}\right|}{c_{i}} G_{j}^{2}\left(y_{j}(t)\right) .
\end{gathered}
$$

It follows that,

$$
\begin{gathered}
\frac{\partial V(y)(t)}{\partial t} \leq\left(1+\left(t-t_{0}\right)^{2}\right)\left\{-2 \sum_{i=1}^{n} y_{i}^{2}(t)+\sum_{i=1}^{n} \sum_{j=1}^{n} \frac{a_{i j}}{c_{i}} y_{i}^{2}(t)\right. \\
+\sum_{i=1}^{n} \sum_{j=1}^{n} \frac{a_{i j}}{c_{i}} F_{j}^{2}\left(y_{j}(t)\right)+\sum_{i=1}^{n} \sum_{j=1}^{n} \frac{\left|b_{i j}\right|}{c_{i}} G_{j}^{2}\left(y_{j}(t)\right)+\frac{1}{\sigma} \lambda_{\max }\left(C^{-1} B Q^{-1} B^{T} C^{-1}\right) \sum_{i=1}^{n} y_{i}^{2}(t) \\
\left.+\frac{2 \epsilon^{*}\left(t-t_{0}\right)}{1+\left(t-t_{0}\right)^{2}} \sum_{i=1}^{n} \frac{1}{c_{i}} y_{i}^{2}(t)\right\}+\sum_{i=1}^{n} \sum_{j=1}^{n}\left[\left(1+\left(t-t_{0}\right)^{2}\right) \sigma \cdot q_{i j}\right. \\
\left.-\left(1+\left(t-\tau(t)-t_{0}\right)^{2}\right) \frac{\left|b_{i j}\right|}{c_{i}}\right] G_{j}^{2}\left(y_{j}(t-\tau(t))\right)
\end{gathered}
$$


We have:

$$
\begin{aligned}
& 2 \sum_{i=1}^{n} \sum_{j=1}^{n} \frac{1}{c_{i}} b_{i j} y_{i}(t) G_{j}\left(y_{j}(t-\tau(t))\right)=2 y^{T}(t) C^{-1} B G(y(t-\tau(t))) \\
& =2 G^{T}(y(t-\tau(t))) B^{T} C^{-1} y(t)=2[G(y(t-\tau(t))) \sqrt{\sigma}]^{T}\left(B^{T} C^{-1} y(t) \frac{1}{\sqrt{\sigma}}\right) \\
& \quad \leq \sigma G^{T}(y(t-\tau(t))) Q G(y(t-\tau(t)))+\frac{1}{\sigma} y^{T}(t) C^{-1} B Q^{-1} B^{T} C^{-1} y(t) \\
& \leq \sigma \sum_{i=1}^{n} \sum_{j=1}^{n} q_{i j} G_{j}^{2}\left(y_{j}(t-\tau(t))\right)+\frac{1}{\sigma} \lambda_{\max }\left(C^{-1} B Q^{-1} B^{T} C^{-1}\right) \sum_{i=1}^{n} y_{i}^{2}(t) .
\end{aligned}
$$

So, from (6) and (7):

$$
\begin{gathered}
\frac{\partial V(y)(t)}{\partial t} \leq\left(1+\left(t-t_{0}\right)^{2}\right)\left[-2+\max _{1 \leq i \leq n}\left\{\frac{1}{c_{i}} \sum_{j=1}^{n} a_{i j}\right\}\right. \\
\left.+\max _{1 \leq j \leq n}\left\{L_{j}^{2} \sum_{i=1}^{n} \frac{a_{i j}}{c_{i}}\right\}+\max _{1 \leq j \leq n}\left\{M_{j}^{2} \sum_{i=1}^{n} \frac{\left|b_{i j}\right|}{c_{i}}\right\}+\frac{1}{\sigma} \lambda_{\max }\left(C^{-1} B Q^{-1} B^{T} C^{-1}\right)+\frac{\epsilon^{*}}{c_{\min }}\right]\|y(t)\|^{2} \\
+\sum_{i=1}^{n} \sum_{j=1}^{n}\left[\left(1+\left(t-t_{0}\right)^{2}\right) \sigma \cdot q_{i j}-\left(1+\left(t-\tau(t)-t_{0}\right)^{2}\right) \frac{\left|b_{i j}\right|}{c_{i}}\right] G_{j}^{2}\left(y_{j}(t-\tau(t))\right) .
\end{gathered}
$$

Then we obtain,

$$
\frac{\partial V(y)(t)}{\partial t}<0
$$

if

$$
\left(1+\left(t-t_{0}\right)^{2}\right) \sigma \cdot q_{i j} \leq\left(1+\left(t-\tau(t)-t_{0}\right)^{2}\right) \frac{\left|b_{i j}\right|}{c_{i}} .
$$

Therefore, it is sufficient that: $\frac{\sigma \cdot q_{i j} c_{i}}{\left|b_{i j}\right|} \leq \frac{\left(1+\left(t-\tau(t)-t_{0}\right)^{2}\right)}{\left(1+\left(t-t_{0}\right)^{2}\right)}$.

Let $u(t)=\frac{1+(t-\tau(t))^{2}}{1+t^{2}}$, next we show for $t \geq 0$

$$
u(t) \geq \frac{\tau^{2}+4-\tau \sqrt{\tau^{2}+4}}{\tau^{2}+4+\tau \sqrt{\tau^{2}+4}} .
$$

First, for $t \in\left[\tau,+\infty\left[\right.\right.$, we have: $u(t) \geq \frac{1+(t-\tau)^{2}}{1+t^{2}}=v(t)$, it is easy to compute that for $t \geq 0$

$$
v_{\min }=v\left(\frac{\tau+\sqrt{\tau^{2}+4}}{2}\right)=\frac{\tau^{2}+4-\tau \sqrt{\tau^{2}+4}}{\tau^{2}+4+\tau \sqrt{\tau^{2}+4}},
$$

also we obtain $v(\tau)>v_{\min }$, that is

$$
\frac{1}{1+\tau^{2}} \geq \frac{\tau^{2}+4-\tau \sqrt{\tau^{2}+4}}{\tau^{2}+4+\tau \sqrt{\tau^{2}+4}}
$$


Second, for $t \in[0, \tau[$, we have:

$$
u(t)=\frac{1+(t-\tau(t))^{2}}{1+t^{2}} \geq \frac{1}{1+\tau^{2}} .
$$

In view of (10) and (11), we obtain that (9) also holds for $t \in[0, \tau[$. Then we obtain that we have proved (9) holds for all $t \in[0,+\infty[$.

We have $\forall k \geq 1$ :

$$
\begin{aligned}
& V(y)\left(t_{k}\right)=\left[1+\left(t_{k}-t_{0}\right)^{2}\right] \sum_{i=1}^{n} \frac{1}{c_{i}} y_{i}^{2}\left(t_{k}\right)+\sum_{i=1}^{n} \sum_{j=1}^{n} \frac{\left|b_{i j}\right|}{c_{i}} \int_{t_{k}-\tau\left(t_{k}\right)}^{t_{k}}\left(1+\left(s-t_{0}\right)^{2}\right) G_{j}^{2}\left(y_{j}(s)\right) d s \\
& =\left[1+\left(t_{k}-t_{0}\right)^{2}\right] y^{T}\left(t_{k}^{-}\right) D_{k} C^{-1} D_{k} y\left(t_{k}^{-}\right)+\sum_{i=1}^{n} \sum_{j=1}^{n} \frac{\left|b_{i j}\right|}{c_{i}} \int_{t_{k}^{-}-\tau\left(t_{k}^{-}\right)}^{t_{k}^{-}}\left(1+\left(s-t_{0}\right)^{2}\right) G_{j}^{2}\left(y_{j}(s)\right) d s \\
& \leq\left[1+\left(t_{k}-t_{0}\right)^{2}\right] \xi_{k} y^{T}\left(t_{k}^{-}\right) y\left(t_{k}^{-}\right)+\sum_{i=1}^{n} \sum_{j=1}^{n} \frac{\left|b_{i j}\right|}{c_{i}} \int_{t_{k}^{-}-\tau\left(t_{k}^{-}\right)}^{t_{k}^{-}}\left(1+\left(s-t_{0}\right)^{2}\right) G_{j}^{2}\left(y_{j}(s)\right) d s \\
& \leq\left[1+\left(t_{k}-t_{0}\right)^{2}\right] \frac{\xi_{k}}{\lambda_{\min }\left(C^{-1}\right)} y^{T}\left(t_{k}^{-}\right) C^{-1} y\left(t_{k}^{-}\right)+\sum_{i=1}^{n} \sum_{j=1}^{n} \frac{\left|b_{i j}\right|}{c_{i}} \int_{t_{k}^{-}-\tau\left(t_{k}^{-}\right)}^{t_{k}^{-}}\left(1+\left(s-t_{0}\right)^{2}\right) G_{j}^{2}\left(y_{j}(s)\right) d s \\
& \leq \max \left\{\frac{\xi_{k}}{\lambda_{\min }\left(C^{-1}\right)}, 1\right\} V\left(t_{k}^{-}\right) .
\end{aligned}
$$

Therefore,

$$
V(y)\left(t_{k}\right) \leq \max \left\{\xi_{k} c_{\max }, 1\right\} V\left(t_{k}^{-}\right)
$$

Then, we have:

$$
\frac{1}{c_{\max }}\left(1+\epsilon^{*}\left(t-t_{0}\right)^{2}\right)\|y(t)\|^{2} \leq V(t) \leq V\left(t_{0}\right) \times \prod_{t_{0}<t_{k} \leq t} \max \left\{\xi_{k} c_{\max }, 1\right\} .
$$

From (5), we have:

$$
\begin{aligned}
\frac{1}{c_{\max }}(1+ & \left.\epsilon^{*}\left(t-t_{0}\right)^{2}\right)\|y(t)\|^{2} \leq V(t) \leq \frac{1}{c_{\min }}\left(1+\epsilon^{*}\left(t-t_{0}\right)^{2}\right)\|y(t)\|^{2} \\
& +\max _{1 \leq i \leq n}\left\{\sum_{j=1}^{n} M_{j}^{2} \frac{\left|b_{i j}\right|}{c_{i}}\right\}\left(\tau(t)+\frac{\left(t-t_{0}\right)^{3}-\left(t-t_{0}-\tau(t)\right)^{3}}{3}\right)\|y(t)\|^{2} .
\end{aligned}
$$

Therefore,

$$
\begin{aligned}
V(t) \leq\left[\frac{1}{c_{\text {min }}}\left(1+\epsilon^{*}\left(t-t_{0}\right)^{2}\right)+\right. & \max _{1 \leq i \leq n}\left\{\sum_{j=1}^{n} M_{j}^{2} \frac{\left|b_{i j}\right|}{c_{i}}\right\}(\tau(t) \\
& \left.\left.+\frac{\left(t-t_{0}\right)^{3}-\left(t-t_{0}-\tau(t)\right)^{3}}{3}\right)\right]\|y(t)\|^{2} .
\end{aligned}
$$

For $t=t_{0}$, we have:

$$
V\left(t_{0}\right) \leq\left[\frac{1}{c_{\min }}+\max _{1 \leq i \leq n}\left\{\sum_{j=1}^{n} M_{j}^{2} \frac{\left|b_{i j}\right|}{c_{i}}\right\}\left(\tau+\frac{\tau^{3}}{3}\right)\right]\|\varphi\|^{2} .
$$


Then,

$$
\frac{1}{c_{\max }}\left(1+\epsilon^{*}\left(t-t_{0}\right)^{2}\right)\|y(t)\|^{2} \leq\left[\frac{1}{c_{\min }}+\max _{1 \leq i \leq n}\left\{\sum_{j=1}^{n} M_{j}^{2} \frac{\left|b_{i j}\right|}{c_{i}}\right\}\left(\tau+\frac{\tau^{3}}{3}\right)\right]\|\varphi\|^{2} \times \prod_{t_{0}<t_{k}<t}\left\{\xi_{k} c_{\max }, 1\right\} .
$$

This implies that:

$\|y(t)\|^{2} \leq\left[\frac{1}{c_{\min }}+\max _{1 \leq i \leq n}\left\{\sum_{j=1}^{n} M_{j}^{2} \frac{\left|b_{i j}\right|}{c_{i}}\right\}\left(\tau+\frac{\tau^{3}}{3}\right)\right] c_{\max }\|\varphi\|^{2} \times \frac{\prod_{t_{0}<t_{k}<t}\left\{\xi_{k} c_{\max }, 1\right\}}{\left(1+\epsilon^{*}\left(t-t_{0}\right)^{2}\right)} \leq \epsilon^{2}$.

Hence, the zero solution of system (1) is uniformly stable.

In view of condition (iv), it is obvious that :

$\limsup \|y(t)\|^{2}=0$, so the equilibrium point of system (1) is also uniformly $t \longrightarrow+\infty$

asymptotically stable and globally asymptotically stable.

Which completes the proof.

If $\prod_{t_{0}<t_{k} \leq t} \max \left\{c_{\max } \cdot \xi_{k}, 1\right\}<\infty$, then we can get the following criterion for stability with $\epsilon^{*}=0$.

Corollary 32. Assume that there is a constant $\sigma>0$ and $n \times n$ positive definite matrix $Q$ such as:

(i) $\sigma \cdot \frac{\max _{i, j} c_{i} \cdot q_{i j}}{\min _{i, j}\left|b_{i j}\right|} \leq \frac{\tau^{2}+4-\tau \sqrt{\tau^{2}+4}}{\tau^{2}+4+\tau \sqrt{\tau^{2}+4}}$

(ii) $\max _{1 \leq i \leq n}\left\{\frac{1}{c_{i}} \sum_{j=1}^{n} a_{i j}\right\}+\max _{1 \leq j \leq n}\left\{L_{j}^{2} \sum_{i=1}^{n} \frac{a_{i j}}{c_{i}}\right\}+\max _{1 \leq j \leq n}\left\{M_{j}^{2} \sum_{i=1}^{n} \frac{\left|b_{i j}\right|}{c_{i}}\right\}$

$+\frac{1}{\sigma} \lambda_{\max }\left(C^{-1} B Q^{-1} B^{T} C^{-1}\right)<2$.

Then, the equilibrium point of system (11) is uniformly asymptotically stable and globally asymptotically stable.

If $\sigma=\frac{\tau^{2}+4-\tau \sqrt{\tau^{2}+4}}{\tau^{2}+4+\tau \sqrt{\tau^{2}+4}}$ and $Q=\frac{1}{60} I d$ in Corollary 32 , then we can get the following criterion for stability.

Corollary 33. Assume that the following conditions are satisfied:

(i) $\frac{\max _{i} c_{i}}{60 \min _{i, j}\left|b_{i j}\right|} \leq 1$

(ii) $\max _{1 \leq i \leq n}\left\{\frac{1}{c_{i}} \sum_{j=1}^{n} a_{i j}\right\}+\max _{1 \leq j \leq n}\left\{L_{j}^{2} \sum_{i=1}^{n} \frac{a_{i j}}{c_{i}}\right\}+\max _{1 \leq j \leq n}\left\{M_{j}^{2} \sum_{i=1}^{n} \frac{\left|b_{i j}\right|}{c_{i}}\right\}$

$+\frac{60}{\sigma} \lambda_{\max }\left(C^{-1} B B^{T} C^{-1}\right)<2$.

Then, the equilibrium point of system (1) is uniformly asymptotically stable and globally asymptotically stable.

\section{Application}

In this section, we present a numerical example to illustrate that our conditions are more feasible than those given in some earlier references [5]. 
Example 41. Consider the two-neuron delayed neural network with impulses as follows:

$$
\left\{\begin{array}{l}
\dot{x_{1}}(t)=-2.5 x_{1}(t)-0.5 f_{1}\left(x_{1}(t)\right)+0.1 f_{2}\left(x_{2}(t)\right) \\
-0.1 g_{1}\left(x_{1}(t-\tau)\right)+0.2 g_{2}\left(x_{2}(t-\tau)\right)-1 \\
\dot{x_{2}}(t)=-2 x_{2}(t)+0.2 f_{1}\left(x_{1}(t)\right)-0.1 f_{2}\left(x_{2}(t)\right) \\
+0.2 g_{1}\left(x_{1}(t-\tau)\right)+0.1 g_{2}\left(x_{2}(t-\tau)\right)+4 \\
\left.\triangle x_{i}\right|_{t=t_{k}}=x_{i}\left(t_{k}\right)-x_{i}\left(t_{k}^{-}\right)=d_{k}^{(i)}\left(x_{i}\left(t_{k}^{-}\right)-\bar{x}_{i}\right) \\
k \in \mathbb{Z}_{+}
\end{array}\right.
$$

where $\tau=0.87$, the activation functions are the following:

$$
f_{1}(x)=f_{2}(x)=g_{1}(x)=g_{2}(x)=0.5(|x+1|-|x-1|)
$$

and $d_{k}^{(1)}=\sqrt{1+\frac{1}{5 k^{2}}}-1, d_{k}^{(2)}=\sqrt{1+\frac{1}{6 k^{2}}}-1, t_{k}=k, k \in \mathbb{Z}_{+}$.

So, the matrix $A, B$ and $C$ are:

$A=\left(\begin{array}{cc}-0.5 & 0.1 \\ 0.2 & 0.1\end{array}\right), B=\left(\begin{array}{cc}-0.1 & 0.2 \\ 0.2 & 0.1\end{array}\right), C=\left(\begin{array}{cc}2.5 & 0 \\ 0 & 2\end{array}\right)$.

By Matlab, we note

$C^{-1} B B^{T} C^{-1}=\left(\begin{array}{cc}0.008 & 0 \\ 0 & 0.0125\end{array}\right)$ so, $\lambda_{\max }\left(C^{-1} B B^{T} C^{-1}\right)=0.0125$.

Considering the activation functions $f_{1}, f_{2}, g_{1}$ and $g_{2}$, we can choose $L_{i}=1$, $M_{i}=1, i=1,2$.

On the other hand, by Mathematica software, we note

$$
\prod_{s=1}^{\infty} \max _{i=1,2}\left(1+d_{s}^{(i)}\right)^{2}=\prod_{s=1}^{\infty} \max _{i=1,2}\left(1+\frac{1}{5 s^{2}}\right)^{2}<1.4
$$

We show the equilibrium point of (16) is uniformly stable and globally asymptotically stable.

From Corollary [33; $\sigma=\frac{\tau^{2}+4-\tau \sqrt{\tau^{2}+4}}{\tau^{2}+4+\tau \sqrt{\tau^{2}+4}} \simeq 0.4297, \frac{1}{\sigma} \simeq 2.3272$. Thus,

$$
\max _{1 \leq i \leq n}\left\{\frac{1}{c_{i}} \sum_{j=1}^{n} a_{i j}\right\}+\max _{1 \leq j \leq n}\left\{L_{j}^{2} \sum_{i=1}^{n} \frac{a_{i j}}{c_{i}}\right\}+\max _{1 \leq j \leq n}\left\{M_{j}^{2} \sum_{i=1}^{n} \frac{\left|b_{i j}\right|}{c_{i}}\right\}+\frac{60}{\sigma} \lambda_{\max }\left(C^{-1} B B^{T} C^{-1}\right)<2 .
$$

Hence, by Corollary 33, the equilibrium point $(-0.2258,1.9548)^{T}$ of system (16) is uniformly asymptotically stable and globally asymptotically stable.

Remark 41. In [5], the authors proved that system (16) is globally asymptotically stable with upperbound of delays $\tau=0.17$. For this example, we additionally get that the equilibrium point of system (16) is uniformly asymptotically stable and globally asymptotically stable with upperbound of delays $\tau=0.87>0.17$. However, the criteria given in [5] are invalid for $(\tau \geq 0.87)$. Therefore, our results are less conservative and more efficient than those given in [5]. 


\section{Conclusion}

In this paper, a class of HNN with delays is considered. We obtain some new criteria ensuring the global and uniform asymptotic stability of the equilibrium point for such system by using the Lyapunov method and linear matrix inequality. Our results show the effects of delay on the stability of HNN. The results here are compared to earlier results. Our criteria are more simpler to verify. An example is given to illustrate the efficiency of the results.

\section{References}

1. Hopfield, J.J.: Neural networks and physical systems with emergent collective computational abilities. Proc. Natl. Acad. Sci. 79, 2554-2558 (1982)

2. Hopfield, J.J.: Neurons with graded response have collective computational properties like those of two-state neurons. Proc. Natl. Acad. Sci. 81, 3088-3092 (1984)

3. Singh, V.: On global robust stability of interval Hopfield neural networks with delays. Chaos Solitons and Fractals 33, 1183-1188 (2007)

4. Huang, H., Cao, J.: On global asymptotic stability of recurrent neural networks with time-varying delays. Applied Mathematics and Computation 142, 143-154 (2003)

5. Zhang, Q., Xu, X.W.J.: Delay-dependent global stability results for delayed Hopfield neural networks. Chaos Solitons and Fractals 34, 662-668 (2007)

6. Liu, B.: Almost periodic solutions for Hopfield neural networks with continuously distributed delays. Mathematics and Computers in Simulation 73, 327-335 (2007)

7. Zhou, J., Xiang, L., Liu, Z.: Synchronization in complex delayed dynamical networks with impulsive effects. Physica A 384, 684-692 (2007)

8. Zhang, Y., Sun, J.: Stability of impulsive neural networks with time delays. Physica A 384, 44-50 (2005)

9. Chen, Z., Ruan, J.: Global stability analysis of impulsive Cohen-Grossberg neural networks with delay. Physica A 345, 101-111 (2005)

10. Xiang, H., Yan, K.M., Wang, B.Y.: Existence and global exponential stability of periodic solution for delayed high-order Hopfield-type neural networks. Physica A 352, 341-349 (2006)

11. Zhang, Q., Wei, X., Xu, J.: Delay-dependent global stability condition for delayed Hopfield neural networks. Nonlinear Analysis 8, 997 (2007)

12. Fu, X.L., Yan, B.Q., Liu, Y.S.: Introduction of Impulsive Differential Systems. Science Press, Beijing (2005)

13. Li, X., Chen, Z.: Stability properties for Hopfield Neural Networks with delays and impulsive perturbations. Nonlinear Analysis: Real World Applications 10, 32533265 (2009)

14. Zong, G., Liu, J.: New Delay-dependent Global Asymptotic Stability Condition for Hopfield Neural Networks with Time-varying Delays. International Journal of Automation and Computing, 415-419 (2009)

15. Liao, X., Chen, G., Sanchez, E.: LMI approach for global periodicity of neural networks with time-varying delays. IEEE Transactions on Circuits Syst. I 49, 1033 (2002)

16. Long, S., Xu, D.: Delay-dependent stability analysis for impulsive neural networks with time varying delays. Neurocomputing 71, 1705-1713 (2008)

17. Chen, A., Cao, J., Huang, L.: An estimation of upperbound of delays for global asymptotic stability of delayed Hopfield neural networks. IEEE Trans Circuits. Syst. I 49, 1028-1032 (2002) 